time and state coupling). Remarkably, the super-adiabatic protocol maintained a fidelity around 0.99 even under parameter variations of up to $100 \%$. The final surprise comes in the time cost of the super-adiabatic protocol it's actually not that much slower than the time-minimal composite-pulse protocol, yet has the combined advantages of high fidelity and parameter robustness.

It will be interesting to see where these new protocols find their utility, and whether the same properties are retained in other physical systems and for multiple qubit gates. In quantum computing it remains to be seen if these control protocols satisfy the time-critical decoherence requirements in the various physical platforms; however, there are schemes that are specifically tailored to adiabatic control where the entire system is evolved to a new ground state $^{6}$, and even hybrid schemes relying on adiabatic quantum gates ${ }^{7}$. No doubt these super-adiabatic protocols will prove useful in other applications in quantum information technology where precise and reliable quantum control is required.
Lloyd C. L. Hollenberg is in the Centre for Quantum Computation and Communication, School of Physics, University of Melbourne,

Victoria 3010, Australia.

e-mail:lloydch@unimelb.edu.au

References

1. Ladd, T. D. et al. Nature 464, 45-53 (2010)

2. Bason, M. G. et al. Nature Phys. 8, 147-152 (2012)

3. Lim, R. \& Berry, M. V. J. Phys. A 24, 3255-3264 (1991)

4. Benhelm, J., Kirchmair, G., Roos, C. F. \& Blatt, R. Nature Phys. 4, 463-466 (2008).

5. Roland, J. \& Cerf, N. J. Phys. Rev. A 65, 042308 (2002)

6. Farhi, E. et al. Science 292, 472-475 (2001)

. Bacon, D. \& Flammia, S. T. Phys. Rev. Lett. 103, 120504 (2009)

\title{
INFORMATION STORAGE
}

\section{Dense bytes from antiferromagnetic bits}

Using magnetism to record information was first demonstrated publicly at the Paris Exposition of 1900. Since the 1960s, it has been the dominant form of digital information storage. In all this time, the magnetic characteristic of the medium in which information is stored has remained the same - it has always been ferromagnetic. But ferromagnetism isn't the only kind of magnetism. Now Sebastian Loth and colleagues have demonstrated that it is also possible to store information in an antiferromagnetic medium (Science 335, 196-199; 2012).

And their results suggest that the number of bits stored per square inch of antiferromagnetic material could be much greater than is currently possible in ferromagnetic materials.

Two ways of improving the storage capacity of magnetic media are to reduce the volume of material needed to reliably record a single bit of information and to reduce the spacing between bits. Individual magnetic bits composed of isolated islands of just a few dozen ferromagnetic atoms have already been demonstrated (Appl. Phys. Lett. 96, 102505; 2010). Reducing the spacing between ferromagnetic bits, however, is more challenging because of the effect that the magnetic fields of neighbouring islands can have on each other.

In contrast, the anti-aligned magnetic moments of antiferromagnetic materials result in no net magnetic moment. Consequently, the distance between antiferromagnetic islands can be drastically reduced without fear of the information stored on one island affecting that of neighbouring islands. The drawback, however, is that antiferromagnetic bits are more difficult to switch, and even more difficult to read, than ferromagnetic bits.

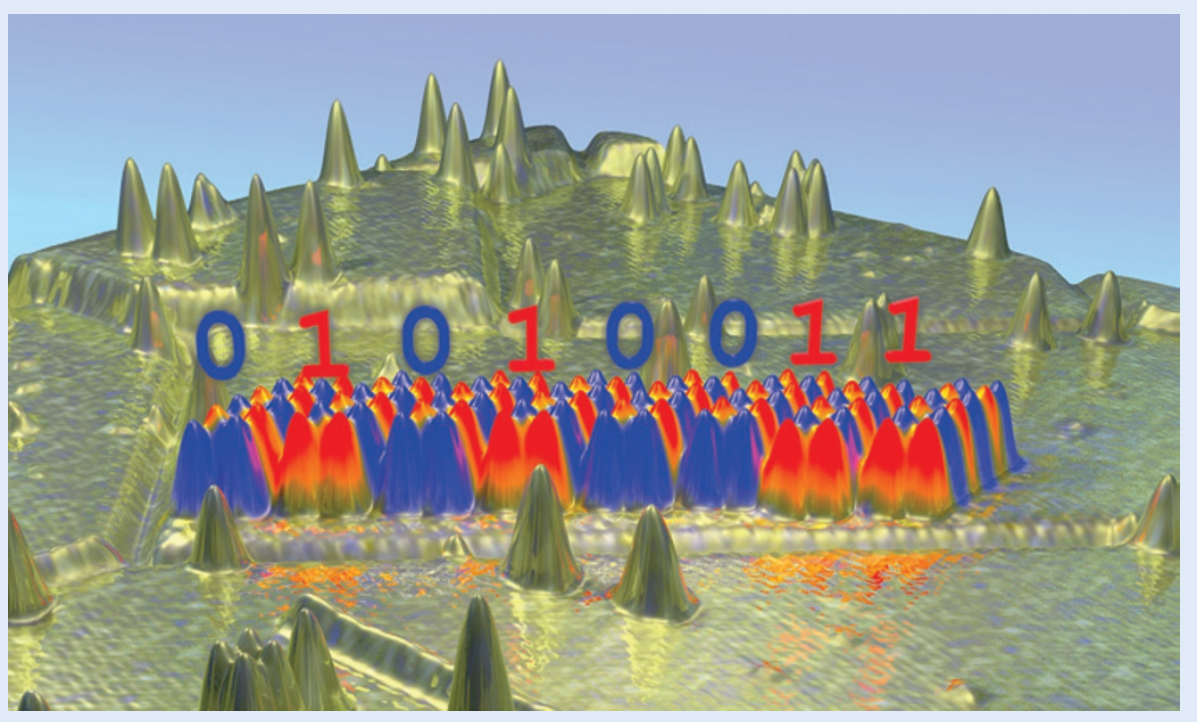

The magnetic storage structures studied by Loth et al. consist of linear arrays of iron atoms deposited one at a time onto a copper nitride surface using a scanning tunnelling microscope (STM). The interaction of the iron atoms with the substrate and with each other causes the axis of their spins to align parallel to the array and causes their directions to alternate antiferromagnetically. The authors find that it takes just eight atoms for the two distinct antiferromagnetic states of such a chain to become stable. They show that they can induce this state to switch by applying a voltage above a certain threshold to the atom at the end of a chain with a magnetized STM tip. And they can determine which state it is in by simply measuring the below-threshold current through the tip.

To demonstrate the information density that might be achievable by such a technique, the authors deposited eight antiferromagnetic chains side by side and stored a byte (eight bits) of information in just 96 irons atoms (pictured). This is much fewer than the hundreds of millions of ferromagnetic atoms needed to store the same information on a conventional hard disk.

The study was carried out at a temperature of $5 \mathrm{~K}$, but the authors expect that increasing the lengths of the chains to 200 atoms could make them stable at room temperature. The need to use a STM to fabricate, write and read these chains means that they are a long way from commercial storage applications, but it does prove the principle that magnetic storage doesn't have to be ferromagnetic. 